\title{
State Evaluation Model of High voltage Switchgear Based on Fuzzy Set-valued Statistical Method and Kernel Vector Space Model
}

\author{
Zhang Xian ${ }^{1}$, Xiong Qian ${ }^{1}$, Cheng Changkui ${ }^{1}$, Li Yuzhe ${ }^{1}$, Liao Liangliang ${ }^{1}$, Shi Shuang ${ }^{1}$, Liu Yobo ${ }^{2}$ \\ ${ }^{1}$ State Grid Tianfu Power Supply Company, Chengdu 610041, China \\ ${ }^{2}$ College of Electric Engineering, Sichuan University, Chengdu 610065, China
}

\begin{abstract}
The traditional high voltage switchgear (HVS) state evaluation model mostly adopts electrical test, live detection and historical data, neglecting the influence of real-time operation data of HVS composition equipment on the state evaluation results. This paper proposes a HVS operation state evaluation model based on fuzzy set-valued statistics method and kernel vector space model based on electrical test data and on-line monitoring data. First of all, according to the components of high voltage switchgear, the operation state of HVS is described and the evaluation index system is established. Secondly, the fuzzy set-valued statistics method is used to construct the mathematical model of evaluation index weight. Then, the kernel vector space model is introduced, and the Gaussian kernel function is used to map the sample to the features of the high-dimensional feature space. The indicator vector of the sample data and the ideal indicator vector of the high-voltage switchgear operation status level standard are defined in the high-dimensional feature space, and the angle-weighted cosine between the two vectors is calculated as the closeness of the sample to the standard status level, and then the high-voltage switchgear operation status level is obtained. Finally, the real data of a power supply company in western China are simulated. The results show that the greater the closeness degree is, the closer the HVS corresponding to the sample is to the normal state, on the contrary, the smaller the closeness degree is, the closer the HVS is to the fault state.
\end{abstract}

\section{Introduction}

With the rapid development of new energy technology, energy storage technology and computer technology, the urban distribution system is highly intelligent [1-2]. As the key equipment of power grid, HVS plays an important role in protecting the safe operation of distribution system lines [3-4]. In recent years, HVS accidents occur frequently in China, which leads to bus power outages and large area power outages caused by main transformer tripping [5-7]. The reasons for the high failure rate of HVS are diversified, especially during the peak summer and windy winter, the equipment insulation aging, operating mechanism failure and high temperature in the cabinet cause burning cabinets, explosions and injuries from time to time, which have a great impact on the production and life of users, and cause significant personal injury and equipment property loss. Therefore, it is an urgent problem to monitor the running state of HVS in real time, make decisions in time, arrange the equipment maintenance plan in advance, and construct the comprehensive evaluation and decision model of HVS operation state.

In recent years, scholars have carried out a lot of research on the condition evaluation of HVS. Literature [8-9] based on fuzzy theory, combined with catastrophe theory and analytic hierarchy process (AHP), the correlation analysis of factors is carried out, and all kinds of experimental data are used to evaluate the state of HVS. The advantage of the model is that the evaluation results are more consistent with the actual situation. The clustering and stratification of the indexes affecting the operation state of switchgear are carried out, the correlation degree is determined by using the normal cloud model, and then the correlation degree is determined by using the normal cloud model. D/S The evidence theory makes a comprehensive evaluation of the overall operation state of the HVS [10]. Literature [11] established a kind of adaptive PSO-LSSVM method, the state evaluation model of multi-dimensional data is used to classify the multi-dimensional historical monitoring data of switchgear and construct the data set, then the least square vector machine algorithm is used to analyse and train the data, and finally the adaptive data is integrated into the model, switchgear state evaluation model with multi-dimensional data is obtained. Literature [12] put forward the concept of ideal interval number, and then the state evaluation model of vibration circuit breaker is established by combining the idea of combination weighting. Compared with the literature [12], the literature [13] considering only the test data of circuit breakers, considering the whole test data and

*Corresponding author's e-mail: zhangx4671@sc.sgcc.com.cn 
historical record of switchgear, a state evaluation method of switchgear based on comprehensive weight and weighted rank sum ratio is established. The model construction is simple and the ability of statistical analysis is strong, and the quantitative evaluation of switchgear state can be realized. The above research results have important guiding significance for switchgear condition maintenance, but they are based on experimental data or historical data, ignore the small but important parameters of HVS operation, and do not consider the electromagnetic field, operating environment and other factors in real time operation of switchgear, and the state evaluation results are lack of integrity. There is little research on the comprehensive evaluation of HVS based on the data of electrical test and real-time monitoring.

In this paper, based on fuzzy set-valued statistics method and kernel vector space model, a mathematical model for evaluating the operation state of HVS is established. Based on the standard of State Power Grid Company, the electrical test index and on-line monitoring index of HVS are determined, and the weight of evaluation index is determined by fuzzy set value statistics method, and then the closeness degree between sample data index and ideal index of standard grade is obtained by using kernel vector space model, which can reflect the real operation of HVS. The proposed method provides a new idea for real-time state evaluation of HVS.

\section{Operation condition Evaluation system of HVS}

\subsection{Running state level description}

The operation state of HVS refers to the state that the complete set of equipment of HVS ensures the safety and stability of power supply on the spot under the influence of electromagnetic field and external environment. Under the harsh electromagnetic field environment or the external environment, the performance of some components of the switchgear gradually decreases, which leads to the abnormal operation state of the switchgear as a whole, which leads to the failure when it is serious. Therefore, considering the electromagnetic field, environment and switchgear structure in substation station, the operation state of switchgear is characterized by normal, attention, abnormal and serious.

The switchgear is in the "normal" state, which indicates that all the components of the switchgear run well, and the parameter value of the component is much less than the attention value of the specification, which is close to the out-of-factory value of the equipment. If there is electrical test data, the data are far less than the attention value. There are also no historical maintenance records, family defect history and other anomalies. In this state, HVS is not need any maintenance.

The HVS is in the "attention" state, indicating that some components of the switchgear are beginning to have small problems. When the component is evaluated separately, its parameter values reach the attention value of the rules, or a certain parameter shows a deterioration trend, which makes the evaluation results in a state of attention, or it may be that there are historical maintenance records or family defect history of switchgear, so it is necessary to pay close attention to the deterioration trend. This state temporarily meets the existing power supply reliability, but cannot ensure the long-term safe operation of the equipment, the maintenance plan should be arranged according to the development trend.

The switchgear is operating in an "abnormal" state, indicating that some components of the switchgear have obvious anomalies or even failures. The parameter value of the component has reached or even exceeded the limit value of the code, or the component index shows a serious deterioration trend, which may cause switchgear failure at any time, so the plan for maintenance should be arranged as soon as possible.

The switchgear is in a "serious" state, indicating that some or all of the component parameters of the switchgear have seriously exceeded the limit value of the regulations, or that the failure of the components can be clearly seen by the naked eye. The HVS is likely to fail at any time and power outage should be arranged immediately for emergency maintenance.

Compilation of reference to National Power Grid Standards [14]. The operation state grade of HVS is divided into normal, attention, abnormal and serious, corresponding to each other. I, II, III and IV Four status levels, the level limits of operational status assessment indicators are shown in Table 1.

Table 1. Operating state evaluation grade boundaries of HVS

\begin{tabular}{ccccccc}
\hline \multirow{2}{*}{ Component } & \multirow{2}{*}{ Evaluation Index } & Unit & \multicolumn{4}{c}{ Grade Boundary } \\
\cline { 3 - 7 } & & & I & II & III & IV \\
\hline \multirow{3}{*}{ Bus } & Temperature & $t_{\text {bus }} /{ }^{\circ} \mathrm{C}$ & 50 & 60 & 70 & 80 \\
\cline { 2 - 7 } & humidity & $H_{\text {bus }} / \%$ & 20 & 25 & 30 & 35 \\
\cline { 2 - 7 } & Dust concentration & $D_{\text {bus }} /\left(\mathrm{mg} / \mathrm{m}^{3}\right)$ & 10 & 15 & 20 & 25 \\
\cline { 2 - 7 } & Arc intensity & $A_{\text {bus }} /\left(\mathrm{nW} / \mathrm{cm}^{2}\right)$ & 6 & 8 & 15 & 30 \\
\cline { 2 - 7 } & Insulation resistance & $R$ bus $/ \mathrm{M} \Omega$ & 2000 & 1000 & 500 & 200 \\
\cline { 2 - 7 } & AC withstand voltage & $V_{\text {bus }} /(\mathrm{kV} / \mathrm{min})$ & 34 & 30 & 25 & $200 \times 10^{-3}$ \\
\hline \multirow{2}{*}{ Breaker } & Vacuum & $P_{\text {bre }} / \mathrm{Pa}$ & $1.33 \times 10^{-2}$ & $1.00 \times 10^{-2}$ & $5.00 \times 10^{-3}$ & $2.00 \times$ \\
\hline
\end{tabular}




\begin{tabular}{|c|c|c|c|c|c|c|}
\hline & Loop resistance & $r_{\text {bre }} / \mu \Omega$ & 20 & 25 & 30 & 35 \\
\hline & Opening time & $T_{\text {ope }} / \mathrm{ms}$ & 40 & 45 & 50 & 55 \\
\hline & Closing time & $T_{\mathrm{clo}} / \mathrm{ms}$ & 55 & 60 & 65 & 70 \\
\hline & Opening coil resistance & $r_{\mathrm{ope}} / \Omega$ & 195 & 205 & 215 & 225 \\
\hline & Closing coil resistance & $r_{\mathrm{clo}} / \Omega$ & 195 & 200 & 205 & 210 \\
\hline & $\begin{array}{c}\text { Different period of } \\
\text { opening }\end{array}$ & $\Delta T_{\text {ope }} / \mathrm{ms}$ & 0.20 & 0.40 & 0.60 & 0.80 \\
\hline & $\begin{array}{c}\text { Different period of } \\
\text { closing }\end{array}$ & $\Delta T_{\mathrm{clo}} / \mathrm{ms}$ & 0.40 & 0.60 & 0.80 & 1.00 \\
\hline & Number of bounces & $S_{\text {bre/ }}$ 次 & 0 & 1 & 2 & 3 \\
\hline & AC withstand voltage & $V_{\text {bre }} /(\mathrm{kV} / \mathrm{min})$ & 42 & 33 & 25 & 20 \\
\hline & Load current & $I_{\mathrm{bre}} / \mathrm{A}$ & 2000 & 2500 & 3000 & 3500 \\
\hline & temperature & $t_{\text {bre }} /{ }^{\circ} \mathrm{C}$ & 40 & 50 & 60 & 70 \\
\hline & humidity & $H_{\text {bre }} / \%$ & 30 & 40 & 50 & 60 \\
\hline & Dust concentration & $D_{\text {bre }} /\left(\mathrm{mg} / \mathrm{m}^{3}\right)$ & 20 & 40 & 80 & 120 \\
\hline & Arc intensity & $A_{\text {bre }} /\left(\mathrm{nW} / \mathrm{cm}^{2}\right)$ & 8 & 15 & 30 & 50 \\
\hline & Opening circuit current & $I_{\text {ope }} / \mathrm{A}$ & 1.20 & 1.00 & 0.80 & 0.60 \\
\hline & Closing circuit current & $I_{\mathrm{clo}} / \mathrm{A}$ & 1.20 & 1.00 & 0.80 & 0.60 \\
\hline & $\begin{array}{c}\text { Energy storage loop } \\
\text { current }\end{array}$ & $I_{\text {sto }} / \mathrm{A}$ & 1.00 & 0.80 & 0.50 & 0.40 \\
\hline & $\begin{array}{c}\text { Cumulative breaking } \\
\text { times }\end{array}$ & $N_{\text {oac }}$ /次 & 20000 & 30000 & 40000 & 50000 \\
\hline & Insulation resistance & $R_{\mathrm{tra}} / \mathrm{M} \Omega$ & 10000 & 8000 & 5000 & 2000 \\
\hline Voltage/Curre & Ratio error & $\lambda_{\text {tra }} / \%$ & 0.50 & 1.00 & 1.50 & 2.00 \\
\hline Transformer & DC Resistance & $r_{\mathrm{tra}} / \Omega$ & 0.60 & 1.00 & 1.60 & 2.40 \\
\hline & AC withstand voltage & $V_{\text {tra }} /(\mathrm{kV} / \mathrm{min})$ & 33 & 27 & 22 & 18 \\
\hline lightning & Leakage current & $I_{\text {arr }} / \mu \mathrm{A}$ & 20 & 30 & 40 & 50 \\
\hline arrester & Insulation resistance & $R_{\text {arr }} / \mathrm{M} \Omega$ & 10000 & 8000 & 5000 & 2000 \\
\hline & temperature & $t_{\mathrm{cab}} /{ }^{\circ} \mathrm{C}$ & 30 & 35 & 40 & 45 \\
\hline & humidity & $H_{\mathrm{cab}} / \%$ & 40 & 50 & 60 & 70 \\
\hline Coblo & Insulation resistance & $R_{\text {cab }} / \mathrm{M} \Omega$ & 50000 & 40000 & 30000 & 20000 \\
\hline Cable & Dust concentration & $D_{\mathrm{cab}} /\left(\mathrm{mg} / \mathrm{m}^{3}\right)$ & 10 & 20 & 40 & 60 \\
\hline & Ultrasonic amplitude & $U H F_{\mathrm{cab}} / \mathrm{dB}$ & 8 & 16 & 30 & 50 \\
\hline & AC withstand voltage & $V_{\text {tra }} /(\mathrm{kV} / \mathrm{min})$ & 17.5 & 15.0 & 13.0 & 10.0 \\
\hline
\end{tabular}

\subsection{Construction of status Evaluation Index system}

Based on the actual field situation and the existing research results, there are many factors that affect the operation state of HVS. In this paper, two big data sets of electrical test and on-line monitoring of switchgear are considered to construct the evaluation index system of operation state of HVS.

- $\quad$ Electrical test index
The HVS runs under the interaction of electromagnetic field and environment, and the failure probability of the component is high, which leads to the abnormal operation and even fault of the switchgear. Electrical preventive test is an important means to sense the state of equipment in advance. The regulation formulates the electrical test index, but some tests are destructive and it is difficult to obtain the actual data. Therefore, this paper will select the more important indicators, as shown in the Figure 1. 


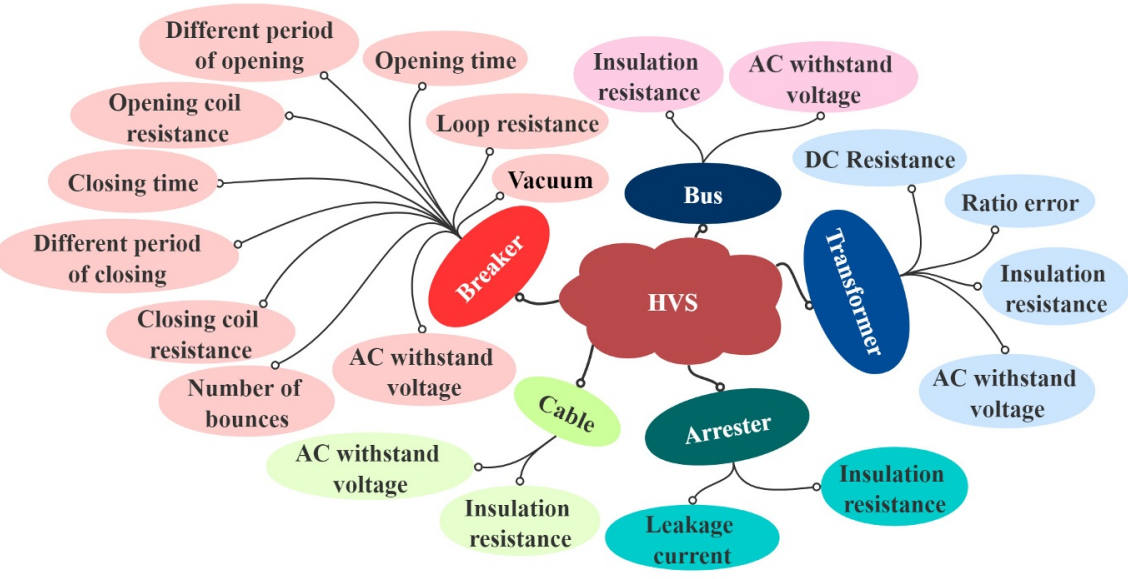

Figure 1. Electrical test indicators of HVS

- Online monitoring indicators

Switchgear power outage in order to carry out electrical prevention test, and some data must be operated switch cabinet to obtain, it is necessary to online monitoring device for data acquisition and storage, as shown in the Figure 2.

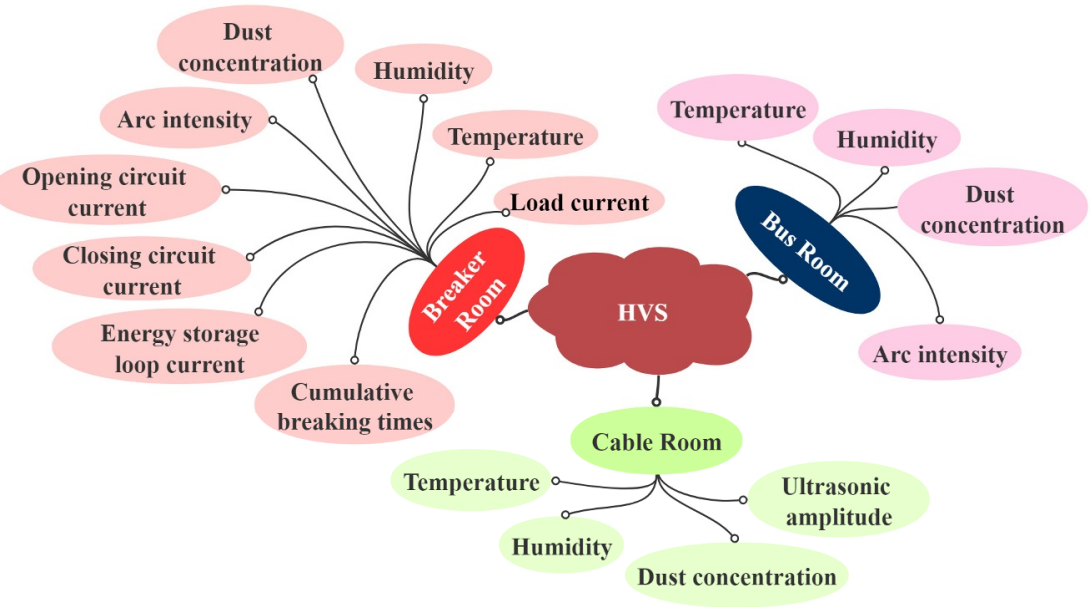

Figure 2. Online monitoring indicators of HVS

\section{Fuzzy set-valued statistics method}

At present, the subjective weight determination method is roughly divided into statistical method and expert scoring method [15]. However, there are many fault types of HVS, lack of on-line monitoring means, and difficult to collect fault sample data. Therefore, the expert scoring method is mainly used to determine the subjective weight. However, the weight of evaluation index has the characteristics of fuzziness and randomness, so it is difficult to describe accurately and quantitatively. It is more in line with the actual situation to give a general description range, that is, the core idea of fuzzy set-valued statistics method [16-17].

Assume that there are $m$ high-voltage switchgear condition assessment indicators, denote by the set $A=$ $\left\{a_{1}, a_{2}, \ldots, a_{m}\right\}$. Indicates that the experts who determine the weight of the evaluation indicators are $n$, denote by the set $Y=\left\{y_{1}, y_{2}, \ldots, y_{n}\right\}$. For the condition evaluation index of HVS $a_{i}$, the index weight ranges assessed by all experts are expressed in turn as follows: $\left[u_{i 1}, w_{i 1}\right],\left[u_{i 2}\right.$, $\left.w_{i 2}\right], \ldots,\left[u_{i n}, w_{i n}\right]$, then the set-valued ranges of all evaluation indicators are shown in the Table 2.

Table 2. The weight interval of index for HVS operating status

\begin{tabular}{ccccc}
\hline \multirow{2}{*}{ Evaluation expert } & \multicolumn{4}{c}{ Operation status evaluation index of HVS } \\
\cline { 2 - 5 } & $a_{1}$ & $a_{2}$ & $\ldots$ & $a_{m}$ \\
\hline$y_{1}$ & {$\left[u_{11}, w_{11}\right]$} & {$\left[u_{21}, w_{21}\right]$} & $\ldots$ & {$\left[u_{m 1}, w_{m 1}\right]$} \\
$y_{2}$ & {$\left[u_{21}, w_{21}\right]$} & {$\left[u_{22}, w_{22}\right]$} & $\ldots$ & {$\left[u_{m 2}, w_{m 2}\right]$} \\
$\ldots$ & $\ldots$ & $\ldots$ & $\ldots$ & $\ldots$ \\
$y_{n}$ & {$\left[u_{n 1}, w_{n 1}\right]$} & {$\left[u_{n 2}, w_{n 2}\right]$} & $\ldots$ & {$\left[u_{m n}, w_{m n}\right]$} \\
\hline
\end{tabular}


To the extent that it is not possible to make a difference between the two groups. To the extent that it is not possible to make a difference between the two groups. This paper is in the process of determining $a_{i}$ When the relative weight is concerned, the undetermined theory is adopted to comprehensively consider the qualification, experience and experience of the experts [18]. Determine that the expert weight is $q_{i}$, after optimization, we get $a_{i}$ Relative weight of $\lambda_{i, r}$, the concrete expression is shown in the following formula.

$$
\lambda_{i, r}=\frac{\sum_{i=1}^{m} q_{i}\left(w_{i n}^{2}-u_{i n}^{2}\right)}{2 \cdot \sum_{i=1}^{m} q_{i}\left(w_{i n}-u_{i n}\right)}
$$

The relative weight is normalized to get the weight vector $R$ of the final evaluation index, is shown in the following formula.

$$
\left\{\begin{array}{l}
R=\left\{r_{1}, r_{2}, \cdots, r_{m}\right\} \\
r_{i}=\lambda_{i, r} / \sum_{i=1}^{m} \lambda_{i, r}
\end{array}\right.
$$

The fuzzy set-valued statistics method determines the weight through the expert experience, although the subjectivity is strong, but the stability is also strong. In practical engineering, there are few changes in the measurement value of the evaluation index, but the importance is high, and the measurement value of the evaluation index changes greatly but the importance is low. It happens that the change of this kind of evaluation index can reflect the state of HVS. Therefore, it is more scientific and reasonable to adjust the weights of the measured values of the index, and to increase the weights of the measured values of the indexes, so that the final evaluation results are more scientific and reasonable. In this paper, entropy method is used [19]. The weight of the initial evaluation index is adjusted dynamically, and the formula is modified, is shown in the following formula.

$$
\left\{\begin{array}{l}
\omega=\frac{1}{2}\left(1+\frac{1}{\ln Z} \sum_{i=1}^{Z} P\left(p_{i j}\right) \cdot \ln P\left(p_{i j}\right)+r_{i}\right) \\
P\left(p_{i j}\right)=p_{i j} / \sum_{i=1}^{Z} p_{i j}
\end{array}\right.
$$

In formula, $Z$ represents the total number of measurements of evaluation indicators, $p_{i j}$ denotes the specific value of the $j^{\text {th }}$ evaluation index in the $i^{\text {th }}$ measurement data.

According to the formula, it can be seen that when evaluating the running state of switchgear, for multiple groups of evaluation index data, the entropy method is used to dynamically correct the initial weight determined above, so as to improve the attention of the evaluation index with a large amount of change.

\section{Kernel vector space model}

Vector Space Model was proposed by scholars Salton et al in the 1970s [20-21], it is widely used in the field of text information retrieval. Later, Chen Jiexi and other scholars are influenced by the idea of kernel clustering, and put forward the Kernel Vector Space Model (KVSM), and in the field of patent classification, the application effect is very good [22]. Therefore, this paper uses KVSM to carry on the comprehensive evaluation to the HVS running state.

Through the nonlinear function, the evaluation samples can be mapped to the high-dimensional feature space, so that the distance between the samples can be maintained enough. The selected kernel function must satisfy Mercer Condition [23]. The existing methods include cross-verification method and empirical method. According to the empirical method, this paper adopts the more popular Gaussian kernel function, and its expression is as follows:

$$
H(a, b)=\exp \left(-\frac{\|a-b\|^{2}}{2 \sigma^{2}}\right)
$$

In formula, $a$ and $b$ represents two spatial vectors respectively. $\sigma$ represents the scale parameters of the Gaussian kernel function.

According to the Enterprise Standard of the State Grid Company 《Q/GDW 1906-2013 Classification Standard for Primary equipment defects of Transmission and Transformation 》 [24], the evaluation value and grade of each index of the evaluation sample are determined. Based on the defect classification of the HVS in the enterprise standard, the standard matrix of the operation state evaluation of the switchgear is established, and the evaluation sample of the input space is mapped to the high dimensional feature space by using the Gaussian kernel function, and the vector space model is established in the high dimensional feature space. The reference status level of switchgear is processed by mathematical method. The vector of $k$ dimensional spatial coordinate system $\boldsymbol{X}_{i}=\left\{x_{i 1}, x_{i 2}, \ldots, x_{i k}\right\}, i$ represents the status level, using a mathematical method to process the samples that need to be evaluated into $k$ dimensional coordinate system vector $\boldsymbol{X}=\left\{x_{1}, x_{2}, \ldots, x_{k}\right\}$. Pick up vector $\boldsymbol{X}_{i}$ and $\boldsymbol{X}$, and then use the Gaussian kernel function to evaluate the sample vector $\boldsymbol{X}_{i}$ and $\boldsymbol{X}$. The standard grade vector is mapped to the sum vector space at the same time, and the angle between the two vectors in the kernel space can be obtained. The cosine angle value whose expression is

$$
\cos \theta_{i}=\frac{H\left(\boldsymbol{X}, \boldsymbol{X}_{i}\right)}{\sqrt{H(\boldsymbol{X}, \boldsymbol{X})} \cdot \sqrt{H\left(\boldsymbol{X}_{i}, \boldsymbol{X}_{i}\right)}}
$$

In order to avoid the weight difference of switchgear state evaluation index, the angle weighted cosine value between the index directed line segment and the ideal index directed line segment is used to measure the closeness between the input sample and the standard sample.

$$
\cos \theta_{i}=\frac{H\left(\omega \boldsymbol{X}, \omega \boldsymbol{X}_{i}\right)}{\sqrt{H(\omega \boldsymbol{X}, \omega \boldsymbol{X})} \cdot \sqrt{H\left(\omega \boldsymbol{X}_{i}, \omega \boldsymbol{X}_{i}\right)}}
$$


After calculating the closeness, the comprehensive evaluation results of the state of HVS are obtained according to the principle of proximity.

\section{Example analysis}

Taking the HVS of a substation of a power supply company in western China as an example, the model is
KYN-28A. The power supply company installed the online monitoring device of HVS in 2019. From 2019 to 2020 , the power supply company carried out many electrical tests on the switchgear in the station, and selected test data one of them are as shown in the Table 3 , the corresponding online device collects data as shown in the Table 4 .

Table 3. Test data of HVS

\begin{tabular}{ccccc}
\hline Index & $\mathrm{D} 1$ & $\mathrm{D} 2$ & $\mathrm{D} 3$ & $\mathrm{D} 4$ \\
\hline$R_{\text {bus }}$ & 10000 & 10000 & 10000 & 10000 \\
$V_{\text {bus }}$ & 34 & 34 & 34 & 34 \\
$P_{\text {bre }}$ & 0.0135 & 0.0132 & 0.0134 & 0.0133 \\
$r_{\text {bre }}$ & 19.8 & 19.4 & 19.5 & 35.5 \\
$T_{\text {ope }}$ & 39.2 & 39.4 & 39.3 & 39.1 \\
$T_{\text {clo }}$ & 53.3 & 68.6 & 54.8 & 55.2 \\
$r_{\text {ope }}$ & 196 & 196 & 197 & 196 \\
$r_{\text {clo }}$ & 195 & 195 & 212.6 & 194 \\
$\Delta T_{\text {ope }}$ & 0.20 & 0.30 & 0.40 & 0.30 \\
$\Delta T_{\text {clo }}$ & 0.10 & 0.20 & 0.10 & 0.30 \\
$S_{\text {bre }}$ & 1 & 2 & 1 & 2 \\
$V_{\text {bre }}$ & 42 & 42 & 42 & 42 \\
$N_{\text {oac }}$ & 3946 & 4026 & 4890 & 6710 \\
$R_{\text {tra }}$ & 10000 & 10000 & 10000 & 10000 \\
$\lambda_{\text {tra }}$ & 0.51 & 0.43 & 0.47 & 0.55 \\
$r_{\text {tra }}$ & 0.48 & 0.52 & 0.53 & 0.49 \\
$V_{\text {tra }}$ & 33 & 33 & 33 & 33 \\
$I_{\text {arr }}$ & 4 & 6 & 7 & 43 \\
$R_{\text {arr }}$ & 10000 & 10000 & 10000 & 10000 \\
$R_{\text {cab }}$ & 50000 & 50000 & 50000 & 50000 \\
$V_{\text {cab }}$ & 17.4 & 17.4 & 17.4 & 17.4 \\
\hline & & & &
\end{tabular}

Table 4. Online monitoring data of HVS

\begin{tabular}{ccccc}
\hline Index & D1 & D2 & D3 & D4 \\
\hline$t_{\text {bus }}$ & 46 & 58 & 49 & 40 \\
$H_{\text {bus }}$ & 22 & 20 & 18 & 19 \\
$D_{\text {bus }}$ & 11 & 12 & 12 & 13 \\
$A_{\text {bus }}$ & 4 & 5 & 3 & 4 \\
$I_{\text {bre }}$ & 2265.5 & 2816.6 & 2316.2 & 2032.7 \\
$t_{\text {bre }}$ & 44 & 78 & 46 & 37 \\
$D_{\text {bre }}$ & 8 & 10 & 8 & 9 \\
$A_{\text {bre }}$ & 3 & 5 & 4 & 4 \\
$I_{\text {ope }}$ & 1.11 & 1.10 & 1.12 & 1.11 \\
$I_{\text {clo }}$ & 1.08 & 1.12 & 0.15 & 1.10 \\
$I_{\text {sto }}$ & 1.01 & 0.98 & 0.99 & 0.98 \\
$t_{\text {cab }}$ & 35 & 38 & 36 & 34 \\
$H_{\text {cab }}$ & 42 & 38 & 43 & 73 \\
$U H F_{\text {cab }}$ & 4 & 4 & 4 & 58 \\
$D_{\text {cab }}$ & 10 & 12 & 11 & 93 \\
\hline
\end{tabular}




\subsection{Only the evaluation results of high voltage test data are taken into account}

According to the experience of high voltage tester in power supply company, some evaluation indexes of high voltage test are selected for simulation analysis. First of all, two senior experts of HVS test and maintenance are invited, and the weight of the selected evaluation index is graded in interval, as shown in the Table 5.

Table 5. The estimation interval of index weight

\begin{tabular}{ccccc}
\hline Index & Expert 1 & Expert 2 & Expert 3 & Expert 4 \\
\hline$R_{\text {bus }}$ & $8-11$ & $8-10$ & $7-10$ & $8-10$ \\
$P_{\text {bre }}$ & $6-9$ & $5-7$ & $5-6$ & $6-7$ \\
$r_{\text {bre }}$ & $5-7$ & $5-6$ & $4-6$ & $5-7$ \\
$T_{\text {ope }}$ & $7-9$ & $8-10$ & $7-9$ & $8-9$ \\
$T_{\text {clo }}$ & $4-5$ & $4-5$ & $5-6$ & $4-6$ \\
$r_{\text {ope }}$ & $6-8$ & $7-8$ & $7-9$ & $7-8$ \\
$r_{\text {clo }}$ & $3-5$ & $4-5$ & $4-5$ & $4-6$ \\
$\Delta T_{\text {ope }}$ & $5-7$ & $5-8$ & $4-7$ & $5-7$ \\
$\Delta T_{\text {clo }}$ & $3-4$ & $4-5$ & $4-5$ & $4-6$ \\
$S_{\text {bre }}$ & $4-6$ & $3-4$ & $4-6$ & $3-5$ \\
$R_{\text {tra }}$ & $3-4$ & $3-5$ & $3-4$ & $4-5$ \\
$\lambda_{\text {tra }}$ & $4-5$ & $3-4$ & $5-6$ & $5-7$ \\
$I_{\text {arr }}$ & $3-6$ & $3-5$ & $3-5$ & $4-6$ \\
$R_{\text {arr }}$ & $5-7$ & $4-7$ & $4-6$ & $4-6$ \\
$R_{\text {cab }}$ & $5-6$ & $6-8$ & $5-7$ & $5-7$ \\
\hline
\end{tabular}

To the extent that it is not possible to make a accordance with the formula (1) to (3) calculate the difference between the two groups. The weight of the four experts is $0.258,0.229,0.246$ and 0.267 , in weight of the dynamically modified evaluation index, as shown in the Table 6.

Table 6. Evaluation index weight after dynamically revised

\begin{tabular}{cccccc}
\hline Weight & $\omega_{1}$ & $\omega_{2}$ & $\omega_{3}$ & $\omega_{4}$ & $\omega_{5}$ \\
\hline Values & 0.0804 & 0.0704 & 0.0612 & 0.0843 & 0.0634 \\
\hline \hline Weight & $\omega_{6}$ & $\omega_{7}$ & $\omega_{8}$ & $\omega_{9}$ & $\omega_{10}$ \\
\hline Values & 0.0807 & 0.0588 & 0.0634 & 0.0583 & 0.0624 \\
\hline \hline Weight & $\omega_{11}$ & $\omega_{12}$ & $\omega_{13}$ & $\omega_{14}$ & $\omega_{15}$ \\
\hline Values & 0.0611 & 0.0665 & 0.0687 & 0.0632 & 0.0572 \\
\hline
\end{tabular}

As we know, samples D1 is close to the normal state

Setting the scale parameter of KVSM is 1.118 into Table 6, the weighted cosine values between five samples and the standard grade are calculated, and the closeness values between the five samples and the normal state of the HVS are shown in the Figure 3.

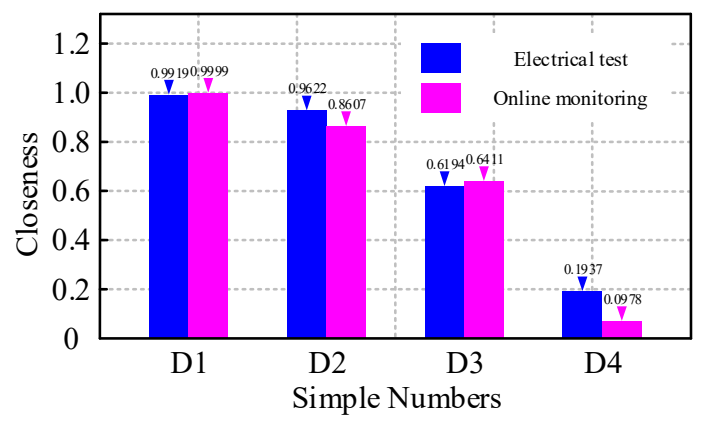

Figure 3. Closeness to the normal state of HVS of the switchgear, and the value approaching 1.0. The degree of closeness to the fault state is about 0 . The errors between all electrical test index data and factory data are within the qualified range, which proves that the sample D1 corresponding switchgear is in a completely "normal" state.

Sample D2 the closeness to the normal state of the switchgear is 0.9622 , the degree of closeness to the fault state is 0.0399 . It is shown that the switchgear is still in a "normal state", which may be due to the deviation of the index data. The maintenance personnel analysed the test data and found that the closing time of the circuit breaker was from the factory $54.8 \mathrm{~ms}$ become $68.6 \mathrm{~ms}$, resulting in a small decrease in closeness, thus judging that the switch cabinet is in an "attention" state.

Sample D3 the closeness to the normal state of the switchgear is 0.6194 , the degree of closeness to the fault state is 0.3806 . The reason may be that there has been a significant change in the value of the index. The inspection was carried out immediately by the 
maintenance personnel, and the resistance of the circuit breaker was detected to be as follows: $212.6 \Omega$, compared with the value of the factory $198.2 \Omega$, it's getting bigger $7.27 \%$. The switching circuit current is too small and the circuit breaker tripping is slow, which may affect the operation of the switchgear, indicating that the switchgear is in an "abnormal" state.

Sample D4 the degree of closeness to the normal state of the switchgear is 0.1937 , the degree of closeness to the fault state is 0.8063 , indicating the sample D4. The corresponding switchgear is in a "fault" state, and the index value seriously exceeds the standard. The maintenance personnel analysed the electrical test data and found that the circuit resistance of the circuit breaker was $35.5 \mu \Omega$, compared with the value of the factory $19.8 \mu \Omega$ has increased $79.29 \%$. The DC leakage current of the arrester is $43 \mu \mathrm{A}$, compared with the value of the factory $3 \mu \mathrm{A}$ increase ten times as much, far beyond the scope of the rules. After the disassembly of the circuit breaker, it is found that the dynamic and static contact in the vacuum interrupter of the circuit breaker is not in good contact, which leads to the excessive contact resistance, which is easy to cause the heating of the contact point and, in serious cases, the deflagration of the switchgear, which seriously affects the safe and stable operation of the power grid. After checking the arrester, it is found that the humidity of the cable room in which the arrester is located has reached the humidity $78 \%$, resulting in a sharp increase in the leakage current.

\subsection{Consider only the evaluation results of online monitoring data}

With the rapid development of sensor technology, online monitoring technology and big data analysis technology, the installation of on-line monitoring device on high-voltage switchgear has made a major breakthrough, and a large number of real-time monitoring data can be obtained. Therefore, based on the online monitoring data, this section carries on the HVS operation condition evaluation. By the same token, two senior experts of HVS test and maintenance are invited to rate the weight of the evaluation index in the interval, as shown in the Table 7 , the weight of the dynamically modified evaluation index is shown in the Table 8 .

Table 7. The estimation interval of index weight

\begin{tabular}{ccccc}
\hline Index & Expert 1 & Expert 2 & Expert 3 & Expert 4 \\
\hline$t_{\text {bus }}$ & $4-5$ & $3-5$ & $4-6$ & $5-6$ \\
$H_{\text {bus }}$ & $5-6$ & $6-7$ & $4-7$ & $5-6$ \\
$D_{\text {bus }}$ & $8-10$ & $7-9$ & $8-11$ & $8-10$ \\
$A_{\text {bus }}$ & $6-8$ & $6-7$ & $5-6$ & $6-7$ \\
$I_{\text {bre }}$ & $6-8$ & $5-6$ & $6-7$ & $5-7$ \\
$t_{\text {bre }}$ & $7-8$ & $6-8$ & $7-9$ & $7-8$ \\
$D_{\text {bre }}$ & $6-8$ & $7-9$ & $7-8$ & $7-8$ \\
$A_{\text {bre }}$ & $5-6$ & $4-6$ & $5-6$ & $5-6$ \\
$I_{\text {ope }}$ & $5-6$ & $4-5$ & $3-4$ & $4-5$ \\
$I_{\text {clo }}$ & $8-11$ & $9-10$ & $9-11$ & $9-10$ \\
$N_{\text {oac }}$ & $6-9$ & $6-7$ & $6-8$ & $7-8$ \\
$I_{\text {sto }}$ & $4-5$ & $3-4$ & $3-5$ & $4-5$ \\
$t_{\text {cab }}$ & $4-5$ & $3-4$ & $3-4$ & $3-5$ \\
$H_{\text {cab }}$ & $7-9$ & $8-9$ & $7-8$ & $8-10$ \\
$D_{\text {cab }}$ & $5-7$ & $6-8$ & $6-7$ & $6-9$ \\
\hline
\end{tabular}

Table 8. Evaluation index weight after dynamically revised

\begin{tabular}{cccccc}
\hline Weight & $\omega_{1}$ & $\omega_{2}$ & $\omega_{3}$ & $\omega_{4}$ & $\omega_{5}$ \\
\hline Values & 0.0611 & 0.0623 & 0.0818 & 0.0683 & 0.0688 \\
\hline Weight & $\omega_{6}$ & $\omega_{7}$ & $\omega_{8}$ & $\omega_{9}$ & $\omega_{10}$ \\
\hline Values & 0.0701 & 0.0691 & 0.0647 & 0.0656 & 0.0824 \\
\hline Weight & $\omega_{11}$ & $\omega_{12}$ & $\omega_{13}$ & $\omega_{14}$ & $\omega_{15}$ \\
\hline Values & 0.0606 & 0.0591 & 0.0582 & 0.0713 & 0.0566 \\
\hline
\end{tabular}

To the extent that it is not possible to make a difference between the two groups. By the same token, the scale parameter $\sigma$ is taken.1.118. The degree of closeness between the sample and the normal state of the HVS is shown in the Figure 4.
As we know, the samples D 1 closeness to the normal state of the switchgear is close to 1.0. The state of switchgear is in a "normal" state.

The sample D2 closeness to the normal state of the switchgear is 0.8607 , the degree of closeness to the fault state of the switchgear is 0.1393 . After analysing the 
monitoring data, it is found that the temperature of the circuit breaker room is $78^{\circ} \mathrm{C}$ (room temperature is $38^{\circ} \mathrm{C}$ ), the load current flowing through the circuit breaker 2816.6A (rated current is $3150 \mathrm{~A}$ ). The preliminary judgment is that the bad contact of the dynamic and static contact of the circuit breaker leads to the temperature rise, and the switchgear is in the state of "attention". According to the planned power outage inspection, the company is indeed caused by the deformation of the contact spring of the circuit breaker.

The sample D3 closeness to the normal state of the switchgear is 0.6411 , the degree of closeness to the fault state of the switchgear is 0.3589 . After in-depth analysis of the on-line monitoring data, it is found that the current of the circuit breaker shuts down and the deviation between the circuit breaker and the normal state is close to that of the normal state $90 \%$. It is preliminarily determined that there is a failure of the opening coil, the circuit breaker has the risk of unable to break, and the switchgear is in an "abnormal" state. According to the plan, the company checked the power outage and found that the opening coil had been burned out.

The sample D4 closeness to the normal state of the switchgear is 0.0978 , the degree of closeness to the fault state of the switchgear is 0.9022 . Based on the analysis of the data, the dust concentration in the cable room has reached $93 \mathrm{mg} / \mathrm{m}^{3}$, the humidity of the cable room is $73 \%$. The amplitude of ultrasonic signal is up to $58 \mathrm{~dB}$. The three indicators exceed the rules, and the switchgear is in a "fault" state. After on-site investigation, the maintenance personnel found that the substation was surrounded by a construction site and the concentration of air dust was $208 \mathrm{mg} / \mathrm{m} 3$. In addition, the ground of the station is low-lying, the water in the cable trench cannot be effectively discharged, resulting in water or moisture seeping into the cable room, the humidity of the cable room is large, the observation window is accompanied by condensation phenomenon, resulting in insufficient insulation distance of HVS and accompanied by discharge phenomenon.

\subsection{Evaluation results taking into account both data}

Based on the previous evaluation results, the operation state of HVS can be obtained by using different data sets. Considering the high voltage test data, the off-line evaluation results are obtained, which cannot reflect the running state. Considering the online monitoring data, the online evaluation results are obtained, which cannot reflect the insulation and mechanical properties of the equipment. Therefore, this paper proposes the fusion of the two data, and proposes a comprehensive evaluation model of HVS operation state based on multiple data sources.

Set the Table 5 And Table 7 Score after data fusion, as shown in the Table 9. Similarly, the scale parameter of KVSM is taken 1.118 , calculate the closeness of the sample and the normal state of the HVS, as shown in the Table 10.

Table 9. The estimation interval of index weight

\begin{tabular}{ccccc}
\hline Index & Expert 1 & Expert 2 & Expert 3 & Expert 4 \\
\hline$R_{\text {bus }}$ & $4-6$ & $4-5$ & $4-5$ & $4-5$ \\
$P_{\text {bre }}$ & $3-5$ & $3-4$ & $3-3$ & $3-4$ \\
$r_{\text {bre }}$ & $3-4$ & $3-3$ & $2-3$ & $3-4$ \\
$T_{\text {ope }}$ & $4-5$ & $4-5$ & $4-5$ & $4-5$ \\
$T_{\text {clo }}$ & $2-3$ & $2-3$ & $3-3$ & $2-3$ \\
$r_{\text {ope }}$ & $3-4$ & $4-4$ & $4-5$ & $4-4$ \\
$r_{\text {clo }}$ & $2-3$ & $2-3$ & $2-3$ & $2-3$ \\
$\Delta T_{\text {ope }}$ & $3-4$ & $3-4$ & $2-4$ & $3-4$ \\
$\Delta T_{\text {clo }}$ & $2-2$ & $2-3$ & $2-3$ & $2-3$ \\
$S_{\text {bre }}$ & $2-3$ & $2-2$ & $2-3$ & $2-3$ \\
$R_{\text {tra }}$ & $2-3$ & $2-2$ & $3-3$ & $3-4$ \\
$\lambda_{\text {tra }}$ & $3-4$ & $3-4$ & $4-5$ & $3-4$ \\
$I_{\text {arr }}$ & $3-4$ & $2-4$ & $2-3$ & $2-3$ \\
$R_{\text {arr }}$ & $3-3$ & $3-4$ & $3-4$ & $3-4$ \\
$R_{\text {cab }}$ & $2-3$ & $2-3$ & $2-2$ & $2-3$ \\
$t_{\text {bus }}$ & $2-3$ & $2-3$ & $2-3$ & $3-3$ \\
$H_{\text {bus }}$ & $3-3$ & $3-4$ & $2-4$ & $3-3$ \\
$D_{\text {bus }}$ & $4-5$ & $4-5$ & $4-6$ & $4-5$ \\
$A_{\text {bus }}$ & $3-4$ & $3-4$ & $3-3$ & $3-4$ \\
$I_{\text {bre }}$ & $3-4$ & $3-3$ & $4-4$ & $3-4$ \\
$t_{\text {bre }}$ & $4-4$ & & & $4-4$ \\
$D_{\text {bre }}$ & $3-4$ & $4-5$ & \\
\hline & & $3-4$ & \\
\hline
\end{tabular}




\begin{tabular}{ccccc}
\hline$A_{\text {bre }}$ & $3-3$ & $2-3$ & $3-3$ & $3-3$ \\
$I_{\text {ope }}$ & $3-3$ & $2-3$ & $2-2$ & $2-3$ \\
$I_{\text {clo }}$ & $4-6$ & $5-5$ & $5-6$ & $5-5$ \\
$N_{\text {oac }}$ & $3-5$ & $3-4$ & $3-4$ & $4-4$ \\
$I_{\text {sto }}$ & $2-3$ & $2-2$ & $2-3$ & $2-3$ \\
$t_{\text {cab }}$ & $2-3$ & $2-2$ & $2-2$ & $2-3$ \\
$H_{\text {cab }}$ & $4-5$ & $4-5$ & $4-4$ & $4-5$ \\
$D_{\text {cab }}$ & $3-4$ & $3-4$ & $3-4$ & $3-5$ \\
\hline
\end{tabular}

Table 10. The estimation interval of index weight

\begin{tabular}{ccccc}
\hline Simple Numbers & D1 & D2 & D3 & D4 \\
\hline Closeness & 0.9999 & 0.9772 & 0.5719 & 0.1853 \\
\hline
\end{tabular}

As we know, the samples D1 and D2 closeness to the normal state of the switchgear is still close to 1.0 that of the switchgear is in a normal state of operation, and the sample D3 closeness to the normal state of the switchgear is 0.5719 . The reason is that the importance of the closing time index in the multi-source data is lower than that of the opening coil resistance index. Although the closing time index changes greatly, this paper perfectly circumvents by dynamically adjusting the index weight, forcing the small change of the opening coil resistance to be able to run the HVS anyway. Similarly, the sample D4 also has a sample D3 similar situation. It can be seen that the condition evaluation of HVS is carried out after the fusion of electrical test data and on-line monitoring data, and the results are more in good agreement with the actual situation.

\section{Conclusion}

Based on the electrical test and on-line monitoring data of switchgear, the comprehensive evaluation model of HVS operation state is established by using fuzzy setvalued statistics method and kernel vector space model in this paper. the conclusions are as follows:

(1) The running state of HVS is described, and a comprehensive evaluation index system of HVS is established.

(2) the fuzzy set-valued statistics method is used to determine the initial weight of the state evaluation index of HVS. In view of the change of some important indexes, the real state of switchgear cannot be reflected. In this paper, the entropy method is used to adjust the initial weight dynamically combined with the test data.

(3) In order to solve the problem that the fuzzy evaluation theory of membership function relies too much on the subjectivity of experts, a comprehensive state evaluation model of HVS is established by using kernel vector space model. In practical engineering, users can select the evaluation index of switchgear according to the actual needs of the field, and realize the comprehensive evaluation of the operation state of HVS from different angles, which provides a novel quantitative analysis idea for power grid operators.

\section{Acknowledgements}

This paper is supported by the Science and Technology Project of State Grid Sichuan Electric Power Company (Project no. SGSC1F00YWJS1900221)

\section{References}

1. Liang Wei, Jin Xiaolong, Mu Yunfei, et al. (2014) Service restoration method to active distribution network with microgrids and electric vehicles[J]. Proceedings of the CSU-EPSA, 26(6): 1-9.

2. Pei Wei, Sheng Kun, Kong Li, et al. (2008) Impact and improvement of distributed generation on distribution network voltage quality[J]. Proceedings of the Chinese Society for Electrical Engineering, 28(13): 152-157.

3. Zhou Xiaoyi, Jeffrey Andle, Wang Xiaofeng, et al. (2019) Feasibility study on installation method of surface acoustic wave ( SAW) sensor circuit breaker contact $\operatorname{arm}[\mathrm{J}]$. High Voltage Apparatus, 55(1): 72-79.

4. Huang Xinbo, Xue Zhipeng, Tian Yi, et al. (2019) Early thermal fault warning strategy of high voltage switch cabinet and its application[J]. Electric Power Automation Equipment, 39(7): 181-187.

5. Pan Changming, Liu Gang, Xiong Ju, et al. (2011) HVS insulation accident's analysis and countermeasures[J]. High Voltage Apparatus, 47(7): 90-93.

6. Yang Zhichun, Le Jian, Jin Chao, et al. (2011) $\mathrm{HV}$ and MV switch cabinet over-temperature supervising system based on gas sensor[J]. Electric Power Automation Equipment, 31(4): 116-119.

7. Ding Jian. (2012) Heating mechanism analysis on metal-enclosed HVS contact with measures to prevent overheating $[\mathrm{J}]$. High Voltage Apparatus, 48(8): 114-117.

8. Xie Jing, Shu Hongchun, Wang Ke, et al. (2014) State evaluation algorithm based on catastrophe 
progression method of HVSs[J]. High Voltage Apparatus, 40(8): 2372-2380.

9. Xie Jing, Shu Hongchun, Wang Ke, et al. (2014) State evaluation based on AHP fuzzy theory of HVSs[J]. High Voltage Engineering, 40(10): 3186-3192.

10. Jia Yanan, Liu Dongming, Sui Huibin. (2017) Comprehensive evaluation for switchgear based on the normal cloud model and D-S evidence theory[J]. High Voltage Apparatus, 53(9): 247252.

11. Shi Xueyan, Zhang Zhousheng, Dong $\mathrm{Na}$, et al. (2019) State evaluation method of multidimensional switchgear data based on adaptive PSO-LSSVM[J]. Water Resources and Power, 37(1): 179-182.

12. Wang Quan, Zhou Yunhai, Yao Junwei, et al. (2019) State evaluation of vacuum circuit breaker based on combination weighting and ideal interval method[J]. High Voltage Apparatus, 55(4): 44-50.

13. Wu Han, Li Shiying, Qi Yanxun, et al. (2020) State evaluation method of high-voltage switchgear based on comprehensive weighting and WRSR[J]. High Voltage Apparatus, 56(2): 47-52.

14. Production and technology department of state grid. (2008) Compilation of regulations and technical standards for equipment condition maintenance[M]. Beijing: China Electric Power Publishing House.

15. Chen Yuehua, Huang Gang. (2017) Improved method of determining comprehensive weight based on rough set theory and AHP $[\mathrm{J}]$. Measurement \& Control Technology, 36(6): 132135.

16. Liu Yunpeng, Xu Ziqiang, Fu Haochuan, et al. (2020) Insulation condition assessment method of power transformer based on improved extension cloud theory with optimal cloud entropy[J]. High Voltage Engineering, 46(2): 397-405.

17. Wang Zhiliang, Wu Bing, Xing Shuren, et al. (2004) Application of fuzzy centralization statistical method in weighted index value of coal mine safety assessment[J]. China Safety Science Journal, 14(1): 71-73.

18. Liao Ruijin, Huang Feilong, Yang Lijun, et al. (2010) Calculation method of power transformer condition assessment index weight using unascertained theory $[\mathrm{J}]$. High Voltage Engineering, 36(9): 2219-2224.

19. Zhang Jiamei, Li Canbing, Peng Minfang, et al. (2018) Vulnerable links analysis based on integrated active power betweenness in active distribution network[J]. Power System Protection and Control, 46(1): 41-48.

20. Wang Cuiyang, Jiang Quanyuan, Tang Yajie, et al. (2019) Fault diagnosis of power dispatching based on alarm signal text mining $[\mathrm{J}]$. Electric Power Automation Equipment, 39(4): 126-132.

21. Chen S, Chen Y, Yuan F, et al. (2018) Establishment of herbal prescription vector space model based on word co-occurrence[J]. The Journal of Supercomputing, 3: 3590-3601.

22. Ding Yuehua, Wen Guihua, GuoWeiqiang. (2005) Patent categorization based on kernel vector space model[J]. Journal of South China University of Technology (Natural Science Edition), 33(8): 5861.

23. Fang Jiayan, Liu Qiao, Wu De, et al. (2018) Smooth support vector machine with similaritybased feature transformation technique and fuzzy C-means clustering[J]. ACTA Electronica Sinica, 46(11): 2714-2724.

24. Q/GDW 1906-2013. Standard for classification of primary equipment defects in transmission and transformation[S]. 